\title{
Caracterización Cualitativa de Poliaminas Libres en Endurecedores de Resinas Epoxídicas del tipo Etilenaminas por Espectroscopia de Resonancia Magnética Nuclear
}

\author{
Filiberto González Garcia \\ Instituto de Ciências Exatas, Universidade Federal de Itajubá \\ Eduardo Miguez, Maria Inês B. Tavares \\ Instituto de Macromoléculas Profa. Eloísa Mano, UFRJ
}

\begin{abstract}
Resumen: La caracterización cualitativa de dos agentes de curado comerciales de resinas epoxídicas del tipo etilenaminas comercializados por la ACROS (U.S.A.) fue realizada usando la espectroscopia de resonancia magnética nuclear de carbono-13. Los productos comerciales corresponden a la trietilentetramina (TETA) y a la tetraetilenpentamina (TEPA). El producto TETA presentó cuatro compuestos diferentes. El compuesto mayoritario correspondió a la trietilentetramina en una concentración de $60 \%$ molar que corresponde a una etilenamina de estructura linear. Los otros tres compuestos, uno de ellos mostró estructura ramificada, y los otros dos compuestos presentaron estructuras cíclicas del tipo piperazina. El producto TEPA mostró cinco compuestos con estructuras semejantes a las encontradas en el producto TETA. El compuesto mayoritario correspondió a la tetraetilenpentamina en una concentración aproximada de 55\% molar. Los resultados encontrados en este trabajo están de acuerdo con la composición cualitativa de los productos semejantes comercializados por la Compañía Química Dow, productos DEH 24 y DEH 26, respectivamente.
\end{abstract}

Palabras-claves: Etilenaminas, polietilen-poliaminas, espectroscopia de resonancia magnética nuclear.

\section{Qualitative Characterization of Free Polyamines in Ethyleneamines Epoxide Hardeners by Nuclear Magnetic Resonance Spectroscopy}

\begin{abstract}
The qualitative characterization of two commercial ethyneamines epoxide hardeners marketed by ACROS (U.S.A.) was carried out by using carbon-13 nuclear magnetic resonance spectroscopy. The products were triethylenetetramine (TETA) and tetraethylenepentamine (TEPA). TETA had four components, the most importante one being triethylenetetramine, an ethyleneamine of lineal structure, in a concentration of $60 \mathrm{~mol} \%$. Another component had ramified structure, while the other two exhibited recurrent structures of the piperazina type. TEPA had five components with similar structures. The major component was tetraethylenepentamine in an approximate concentration of $55 \mathrm{~mol} \%$. These results agree with the composition of similar products marketed by Dow Chemical Company, namely DEH 24 and DEH 26, respectively.
\end{abstract}

Keywords: Ethyleneamines, polyethylene-polyamines, nuclear magnetic resonance spectroscopy.

\section{Introducción}

Una familia de compuestos químicos comúnmente utilizadas como endurecedores o como agentes de curado para el procesamiento de las resinas epoxídicas, son las llamadas indistintamente polietilen-poliaminas o etilenaminas. Las etilenaminas utilizadas como agentes de curado de las resinas epoxídicas son endurecedores del tipo amina alifática, y su acción como endurecedor consiste en el proceso de transformación de las moléculas monoméricas de la resina y del agente de curado en un material termofijo a través de reacciones de polimerización, las que son conocidas comúnmente como reacción de reticulación o como reacción de curado.
La polimerización de las resinas epoxídicas utilizando polietilen-poliaminas como co-monómeros está caracterizada por sucesivas reacciones de adición de los átomos de hidrógenos activos del tipo amina primaria y secundaria presentes en la polietilen-poliamina, a los grupos epoxídicos de la resina. De esta manera son formados bloques de unión que contienen estos dos compuestos formando así una red con estructura tridimensional debido a la naturaleza polifuncional de los monómeros. En este caso, la red formada se denomina del tipo epoxi-poliamina, o de manera más generalizada del tipo epoxi-amina. En la reacción se utilizan relaciones estequiométricas, o casi estequiométricas, entre los grupos funcionales, o sea, entre los átomos de

Autor para correspondência: Filiberto González Garcia, Departamento de Física e Química, Instituto de Ciências Exatas, Universidade Federal de Itajubá (UNIFEI), Campus Prof. José Rodrigues Seabra, Avenida BPS 1303, Bairro Pinheirinho, CEP: 37500-903, Itajubá, MG, Brasil. E-mail: fili@unifei.edu.br. 
hidrógenos activos del tipo amina y los grupos epoxídicos, y no se necesita de la adición de catalizadores para que la reacción ocurra a la temperatura ambiente.

Las etilenaminas son una familia de compuestos químicos de fórmula general $\mathrm{H}_{2} \mathrm{~N}\left(\mathrm{CH}_{2} \mathrm{CH}_{2} \mathrm{NH}\right)_{n} \mathrm{CH}_{2} \mathrm{CH}_{2} \mathrm{NH}_{2}$, donde los compuestos más utilizados como endurecedores de resinas epoxídicas son los compuestos de baja masa molecular; dietilentriamina (DETA) $\operatorname{con} \mathrm{n}=1$, trietilentetramina (TETA) con $\mathrm{n}=2$, y tetraetilenpentamina (TEPA) $\operatorname{con} \mathrm{n}=3^{[1]}$. La Compañía Química Dow abrió el camino para el desarrollo de las etilenaminas en 1935, cuando esta compañía lanzó la primera producción de este tipo de compuestos con fines comerciales, y ha sido el líder mundial de este negocio desde entonces. Los compuestos son sintetizados a partir de dicloroetano utilizando procesos catalíticos de aminación reductiva. Las etilenaminas comerciales son mezclas de poliaminas polifuncionales, de baja o de alta masa molecular. Estos compuestos contienen cadenas etilénicas enlazadas químicamente a átomos de nitrógeno del tipo amina. El número de cadenas carbonadas enlazadas químicamente al átomo de nitrógeno, origina que existan átomos de nitrógeno del tipo amina primaria, secundaria y terciaria, y consecuentemente existan compuestos con estructuras químicas lineales, ramificadas y cíclicas (del tipo piperazina) ${ }^{[2]}$. La composición cualitativa y cuantitativa de la mezcla del producto modifica la reactividad de la etilenamina con la resina epoxídica. Muda la concentración del producto en la mezcla con la resina, para obtener la relación estequiométrica y altera el valor del equivalente de hidrógenos activos del tipo amina ${ }^{[3]}$, además de afectar la funcionalidad del producto $^{[4]}$. Esto motiva que sea necesaria la caracterización cualitativa y cuantitativa de la composición de este tipo de productos comerciales para una correcta utilización de las etilenaminas en las formulaciones epoxídicas.

La caracterización cualitativa y cuantitativa de las poliaminas lineales de baja masa molecular $(n=0,1,2$ y 3$)$ ha sido realizada por diferentes métodos cromatográficos ${ }^{[5-7]}$. Un esquema aditivo para el cálculo de los corrimientos químicos de polietilen-poliaminas comerciales sintetizadas en la antigua U.S.S.R., fue divulgado usando la espectroscopia de resonancia magnética nuclear de carbono-13 (RMN $\left.{ }^{13} \mathrm{C}\right)$. Este esquema aditivo de cálculo estuvo fundamentado en la utilización de compuestos modelo de estructura lineal (etilendiamina, trietilentetramina) y de estructura cíclica (piperazina), así como por la utilización de compuestos modelo de estructura ramificada (polietilen-iminas) ${ }^{[8]}$. Sin embargo, en la literatura no aparecen trabajos científicos donde, se divulga la composición de las etilenaminas comerciales, ya que la información relativa a la síntesis y la composición de los productos comerciales, está protegida por patentes de las compañías químicas productoras. Esto nos motivó a realizar un trabajo anterior que fue publicado en revista regional ${ }^{[9]}$. En el cual fue caracterizada una polietilen-poliamina de baja masa molecular, existente en Cuba, denominada 720Y. En ese trabajo fueron utilizadas técnicas de resonancia magnética nuclear (RMN ${ }^{1} \mathrm{H}$ y ${ }^{13} \mathrm{C}$ ), técnicas de cromatografía convencional (Columna y TLC), además de utilizar técnicas combinadas de cromatografía gaseosa acoplada a espectrometría de masas (CG-EM).

La experiencia adquirida en aquel trabajo, determinó que la técnica escogida para este trabajo fuera la espectroscopia de resonancia magnética nuclear de carbono-13 $\left(\mathrm{RMN}{ }^{13} \mathrm{C}\right)$, considerando que está es una técnica no destructiva y altamente informativa con relación a la caracterización estructural, además que no necesitar de ningún tratamiento previo de la muestra. Otro motivo fue la posibilidad de utilizar los datos reportados en la literatura ${ }^{[8,9]}$, para realizar las asignaciones correspondientes a los diferentes fragmentos estructurales de etilenaminas, lineales, ramificados y cíclicos, y con esto proponer la estructura de los compuestos existentes. En este trabajo fue realizada la determinación cualitativa de los componentes de dos endurecedores comerciales de resinas epoxídicas del tipo etilenaminas de baja masa molecular, la trietilentetramina (TETA) y la tetraetilenpentamina (TEPA) comercializados pela ACROS (U.S.A.), utilizando la RMN ${ }^{13} \mathrm{C}$. En las condiciones instrumentales utilizadas fue posible determinar la concentración semi-cuantitativa y la concentración aproximada del compuesto mayoritario en cada producto, respectivamente.

\section{Experimental}

\section{Materiales}

Dos endurecedores del tipo polietilen-poliaminas de grado técnico, la trietilentetramina (TETA, 60\%) y la tetraetilenpentamina (TEPA, técnica), comercializados por la ACROS (U.S.A.) fueron utilizados sin tratamiento previo.

\section{Métodos}

Los espectros de resonancia magnética nuclear de carbono-13 (RMN ${ }^{13} \mathrm{C}$ ) fueron obtenidos en un equipo VARIAN Mercury-300 a $75.4 \mathrm{MHz}$, utilizando como solvente el $\mathrm{CDCl}_{3}$ a la temperatura ambiente. Los corrimientos químicos fueron reportados en ppm referidos al TMS como referencia interna. Los espectros de $\mathrm{RMN}{ }^{13} \mathrm{C}$ con desacoplamiento protónico se registraron con los siguientes parámetros: tiempo de adquisición 1,5 s; anchura espectral $18 \mathrm{kHz}$; pulsos $\left(90^{\circ}\right) 35 \mu \mathrm{s}$, número de transientes 4000 , intervalo entre los pulsos (dl) 1s.

\section{Resultados y Discusión}

\section{Caracterización estructural del producto trietilentetramina (TETA)}

En la Figura 1 se muestra el espectro de RMN ${ }^{13} \mathrm{C}$ del producto trietilentetramina (TETA) comercializado por la ACROS (U.S.A.) en la región de 37.0 a 62.0 ppm, donde se incluyen las asignaciones de las señales. Estas asignaciones fueron apoyadas por datos reportados en la literatura ${ }^{[8,9]}$. En 


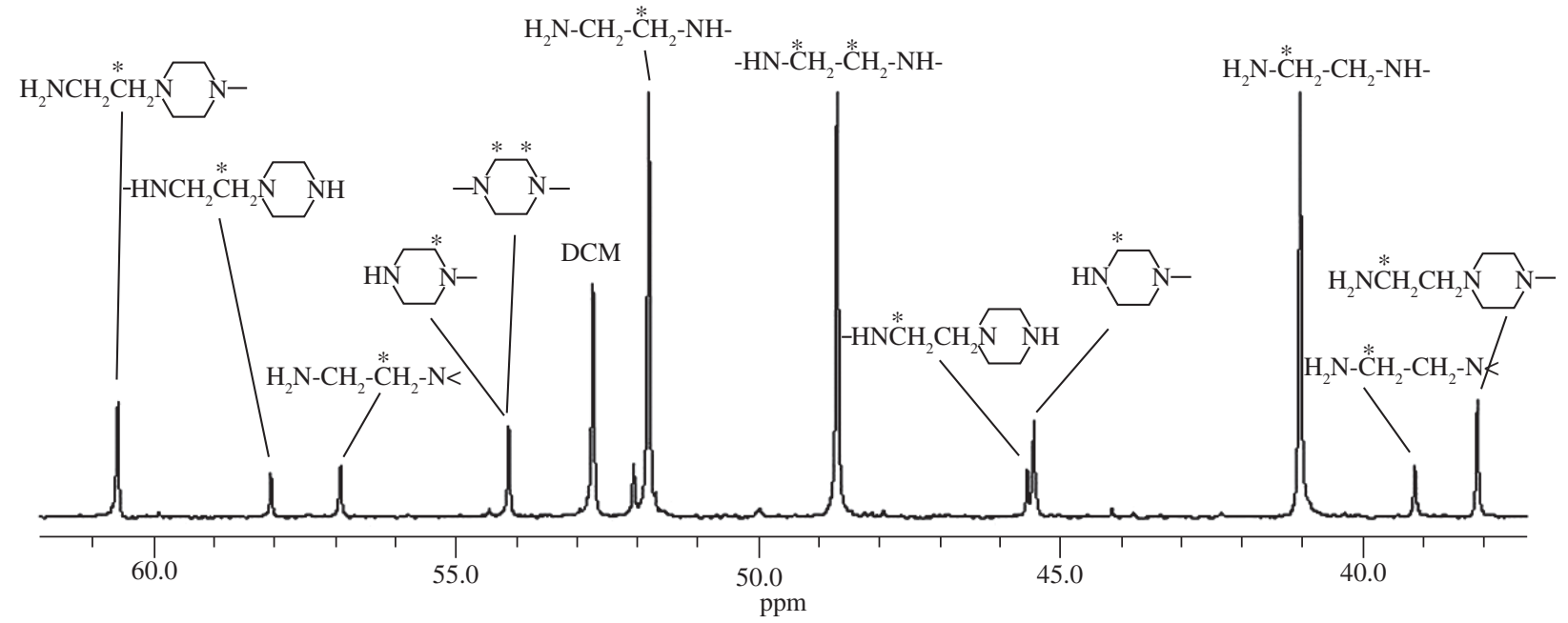

Figura 1. Espectro de RMN ${ }^{13} \mathrm{C}$ del producto trietilentetramina (TETA) comercializado por la ACROS (U.S.A.). DCM, corresponde a la señal del diclorometano en 52.7 ppm, impureza detectada en el producto.

la Tabla 1 se muestran los resultados del análisis de RMN ${ }^{13} \mathrm{C}$, se comparan con los datos de la literatura y con los datos calculados en este trabajo por el empleo de tablas ${ }^{[10]}$. Los datos reportados en la Tabla 1 están de acuerdo con los datos publicados en la literatura para este tipo de compuestos ${ }^{[8]}$. Así como otros datos también divulgados, por la combinación de técnicas cromatográficas y espectroscópicas ${ }^{[9]}$ realizadas durante la caracterización de un endurecedor, denominado $720 Y$, que confirman este análisis. Ya que para este endurecedor denominado $720 Y$, fue confirmado que su composición estaba constituida por un compuesto mayoritario, la trietilentetramina $(62 \%$, molar) y una mezcla de polietilen-poliaminas como las mostradas en la Tabla 2.
La coincidencia de los valores experimentales de este producto comercializado por la ACROS (U.S.A.), y los encontrados anteriormente para el endurecedor $720 \mathrm{Y}^{[9]}$ indicaron que la composición cualitativa de este producto TETA debe ser semejante a la encontrada en el endurecedor 720Y, donde fueron encontradas cinco tipos de etilenaminas polifuncionales. Los compuestos tienen en su estructura química carbonos metilénicos que corresponden a siete fragmentos estructurales como aparece mostrado en la Tabla 1 (fragmentos A, B, $\mathrm{C}, \mathrm{D}, \mathrm{E}, \mathrm{H}$ e I). Estos fragmentos estructurales pueden ser relacionados a cinco estructuras químicas como las mostradas en la Tabla 2 (compuestos 1, 2, 3, 4 y 5).

Tabla 1. Análisis del espectro de $\mathrm{RMN}{ }^{13} \mathrm{C}$ del producto TETA. Corrimientos químicos $(\delta \mathrm{ppm})$.

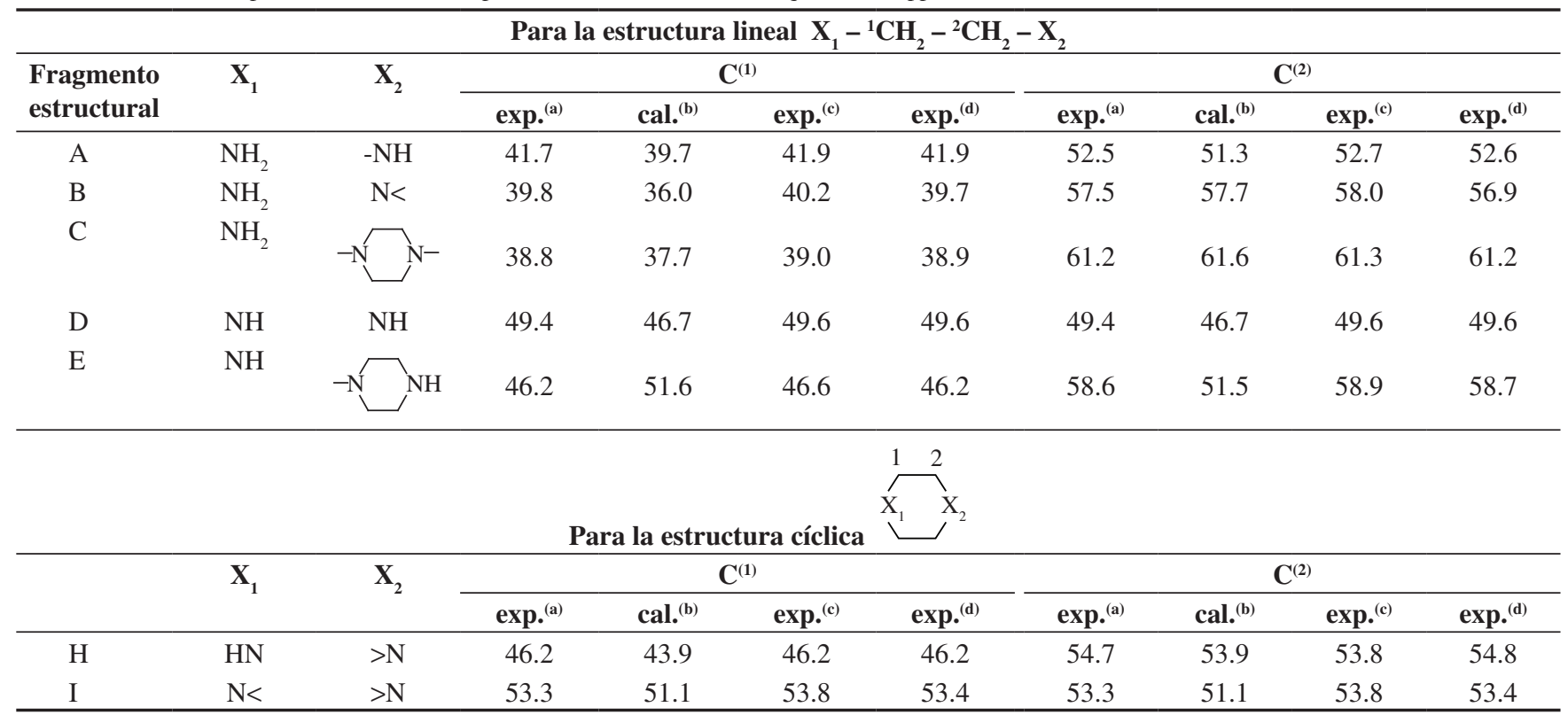

a) Valores experimentales para el producto TETA (ACROS, U.S.A.); b) valores calculados según la referencia 10; c) valores encontrados en la referencia 8; y d) valores encontrados en González Garcia et $\mathrm{al}^{\left[{ }^{[9]}\right.}$. 
Tabla 2. Composición cualitativa del endurecedor $720 \mathrm{Y}$.

\begin{tabular}{|c|c|c|}
\hline 1) & $\mathrm{H}_{2} \mathrm{~N}-\left(\mathrm{CH}_{2}\right)_{2}-\mathrm{NH}-\left(\mathrm{CH}_{2}\right)_{2}-\mathrm{NH}_{2}$ & Dietilentriamina (DETA) \\
\hline 2) & $\mathrm{H}_{2} \mathrm{~N}-\left(\mathrm{CH}_{2}\right)_{2}-\mathrm{NH}-\left(\mathrm{CH}_{2}\right)_{2}-\mathrm{NH}-\left(\mathrm{CH}_{2}\right)_{2}-\mathrm{NH}_{2}$ & Trietilentetramina (TETA) \\
\hline 3) & {$\left[\mathrm{H}_{2} \mathrm{~N}-\left(\mathrm{CH}_{2}\right)_{2}-\right]_{3}-\mathrm{N}$} & Tris-(2-aminoetil)amina (TAEA) \\
\hline 4) & $\mathrm{H}_{2} \mathrm{~N}-\left(\mathrm{CH}_{2}\right)_{2}-\mathrm{N}$ & $4,4^{\prime}$ aminoetilpiperazina $\left(4,4^{\prime} \mathrm{AEP}\right)$ \\
\hline 5) & $\mathrm{N}-\left(\mathrm{CH}_{2}\right)_{2}-\mathrm{NH}-\left(\mathrm{CH}_{2}\right)_{2}-\mathrm{NH}_{2}$ & Dietilendiaminopiperazina (DEDAP) \\
\hline
\end{tabular}

Los compuestos 1 y 2 mostrados en la Tabla 2 contienen átomos de nitrógeno del tipo amina primaria y secundaria, los que están enlazados químicamente de manera alternada a dos y a tres cadenas etilénicas, respectivamente de manera que existen átomos de nitrógeno del tipo amina primaria en los extremos de cada compuesto. Estos compuestos contienen en común fragmentos estructurales del tipo lineal (fragmentos A; y A y D, respectivamente ver Tabla 1). En este caso nos referimos a la dietilentriamina (DETA) y a la trietilentetramina (TETA). En este producto es esperado que el compuesto mayoritario sea la TETA. Esto quedó evidente en el espectro de la Figura 1 por la mayor intensidad relativa de las señales correspondientes a este compuesto cuando comparadas a las intensidades relativas de las otras señales que aparecen en el espectro (señales en 41.7, 52.5 y 49.4 ppm,). Sin embargo, estos valores experimentales no excluyen la presencia de dietilentriamina en la composición de este producto.

El compuesto 3 mostrado en la Tabla 2 presenta en su estructura química un átomo de nitrógeno del tipo terciario, como consecuencia de la existencia de tres cadenas etilénicas enlazadas químicamente a un átomo de nitrógeno. En este compuesto, cada cadena etilénica presenta un átomo de nitrógeno del tipo amina primaria en el otro extremo de la cadena. Nos referimos a la tris-(2-aminoetil)amina (TAEA), que corresponde a una etilenamina con estructura ramificada (fragmento B, Tabla 1). Los otros dos compuestos (4 y 5 Tabla 2), son también etilenaminas polifuncionales, las que presentan en su estructura química de forma diferente átomos de nitrógeno del tipo amina primario, secundario y terciario. Sin embargo, tienen en común la estructura cíclica del tipo piperazina. Nos referimos a la 4,4' aminoetilpiperazina (4,4'AEP) que es una piperazina di-sustituida de manera simétrica, y a la dietilendiaminopiperazina (DEDAP) que corresponde a una piperazina mono-sustituida. En este caso, los dos compuestos presentan una combinación de fragmentos estructurales del tipo lineal y cíclico del tipo piperazina (fragmentos C y I; y H, E y A, respectivamente ver Tabla 1).

La concentración semi-cuantitativa de trietilentetramina (TETA) en el producto comercial de la ACROS (U.S.A.) fue de $60 \%$ molar. Este resultado fue obtenido a partir de la relación entre las áreas relativas de las señales en 41.7, 52.5 y $49.4 \mathrm{ppm}$, correspondientes a los carbonos metilénicos de los fragmentos estructurales del tipo; - $\mathrm{HN}-\mathrm{CH}_{2}-\mathrm{CH}_{2}-\mathrm{NH}_{2},-\mathrm{HN}-\mathrm{CH}_{2}-\mathrm{CH}_{2}-\mathrm{NH}_{2}$, y $-\mathrm{HN}-\mathrm{CH}_{2}-\mathrm{CH}_{2}-\mathrm{NH}-$, respectivamente en la trietilentetramina, en relación a todas las áreas de las señales del espectro. Este resultado coincide con el valor suministrado por el fabricante, lo que indicó que la concentración de dietilentriamina (DETA), si existe en la composición de este producto es muy pequeña, pues no afecta la concentración de trietilentetramina proporcionada por el fabricante.

Estos resultados indicaron que la composición cualitativa fundamental del producto TETA comercializado por la ACROS (U.S.A.) es una mezcla de cuatro tipos de etilenaminas polifuncionales: un compuesto de estructura lineal, la trietilentetramina (TETA), que es el compuesto mayoritario, un compuesto con estructura ramificada, la tris-(2-aminoetil) amina (TAEA), y dos compuestos con estructuras cíclicas; la 4,4' aminoetilpiperazina (4,4'AEP) y la dietilendiaminopiperazina (DEDAP). Esta información coincide con lo divulgado por la Compañía Química Dow para su producto similar de trietilentetramina (TETA) (producto DEH 24) ${ }^{[2]}$.

\section{Caracterización estructural del producto tetraetilenpentamina (TEPA)}

La Figura 2 muestra el espectro de RMN ${ }^{13} \mathrm{C}$ del producto técnico tetraetilenpentamina (TEPA) comercializado por la ACROS (U.S.A.) en la región de 37.0 a 62.0 ppm, donde están incluidas las asignaciones de las señales, las que fueron apoyadas por datos reportados en la literatura ${ }^{[8,9]}$. En la Tabla 3 se muestran los resultados del análisis de $\mathrm{RMN}{ }^{13} \mathrm{C}$ de este producto. En la Tabla se encuentra un buen acuerdo entre los valores experimentales, los calculados en el trabajo ${ }^{[10]}$ y los reportados en la literatura. Los datos espectroscópicos corresponden a nueve fragmentos estructurales de etilenaminas (fragmentos A, B, C, D, E, F, G, H e I). A partir de esta información estructural pueden ser propuestas fundamentalmente cinco tipos de etilenaminas polifuncionales diferentes, con estructuras semejantes a los compuestos propuestos en el producto trietilentetramina (TETA). Estas estructuras químicas eran esperadas, debido a que la tetraetilenpentamina presenta en su estructura un grupo etilenamina adicional 


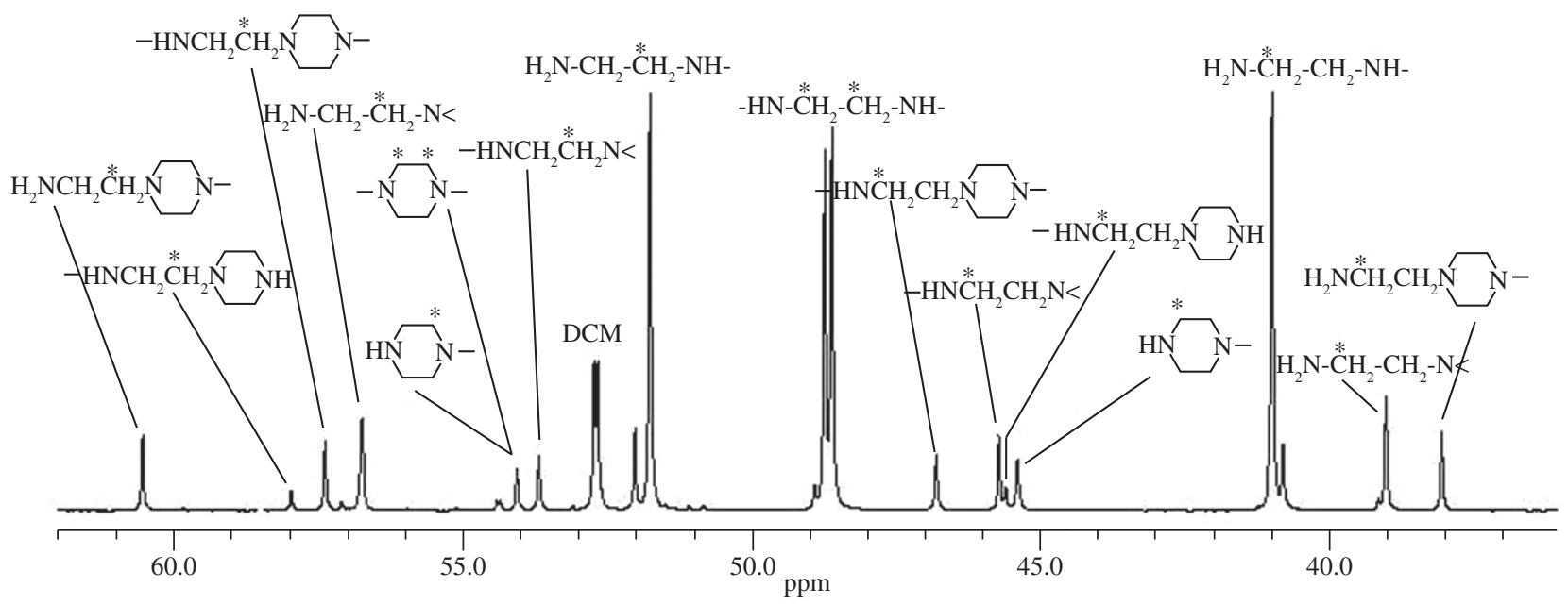

Figura 2. Espectro de RMN ${ }^{13} \mathrm{C}$ del producto tetraetilenpentamina (TEPA) comercializado por la ACROS (U.S.A.). DCM, corresponde a la señal del diclorometano en 52.7 ppm, impureza detectada en el producto.

$\left(>\mathrm{N}-\mathrm{CH}_{2}-\mathrm{CH}_{2}-\mathrm{N}<\right.$ ), cuando comparado a la estructura química de la trietilentetramina. Este comportamiento puede ser resultado del propio proceso de síntesis que es utilizado para la obtención de estos dos productos con estructuras químicas muy semejantes.

En este producto comercial TEPA es esperado que exista grande semejanza entre los valores experimentales del compuesto tetraetilenpentamina, cuando comparado a los valores experimentales encontrados para el compuesto trietilentetramina, debido a la semejanza entre las estructuras químicas de estos dos compuestos, como fue mencionado anteriormente.
Esto fue evidente en los valores experimentales encontrados para el producto técnico de tetraetilenpentamina. No obstante, la señal en $48.6 \mathrm{ppm}$ en este espectro aparece en forma de doblete en 48.7 y 48.6 ppm (Figura 2). Las señales en $40.9,51.7,48.7$ y $48.6 \mathrm{ppm}$, corresponden a fragmentos estructurales del tipo lineal (fragmentos A y D, Tabla 3), los que justifican la presencia de la tetraetilenpentamina en la composición de este producto. Sin embargo, estos valores experimentales no excluyen la presencia de trietilentetramina en la composición de este producto. La señal en forma de

Tabla 3. Análisis del espectro de $\mathrm{RMN}{ }^{13} \mathrm{C}$ del producto TEPA. Corrimientos químicos $(\delta \mathrm{ppm})$.

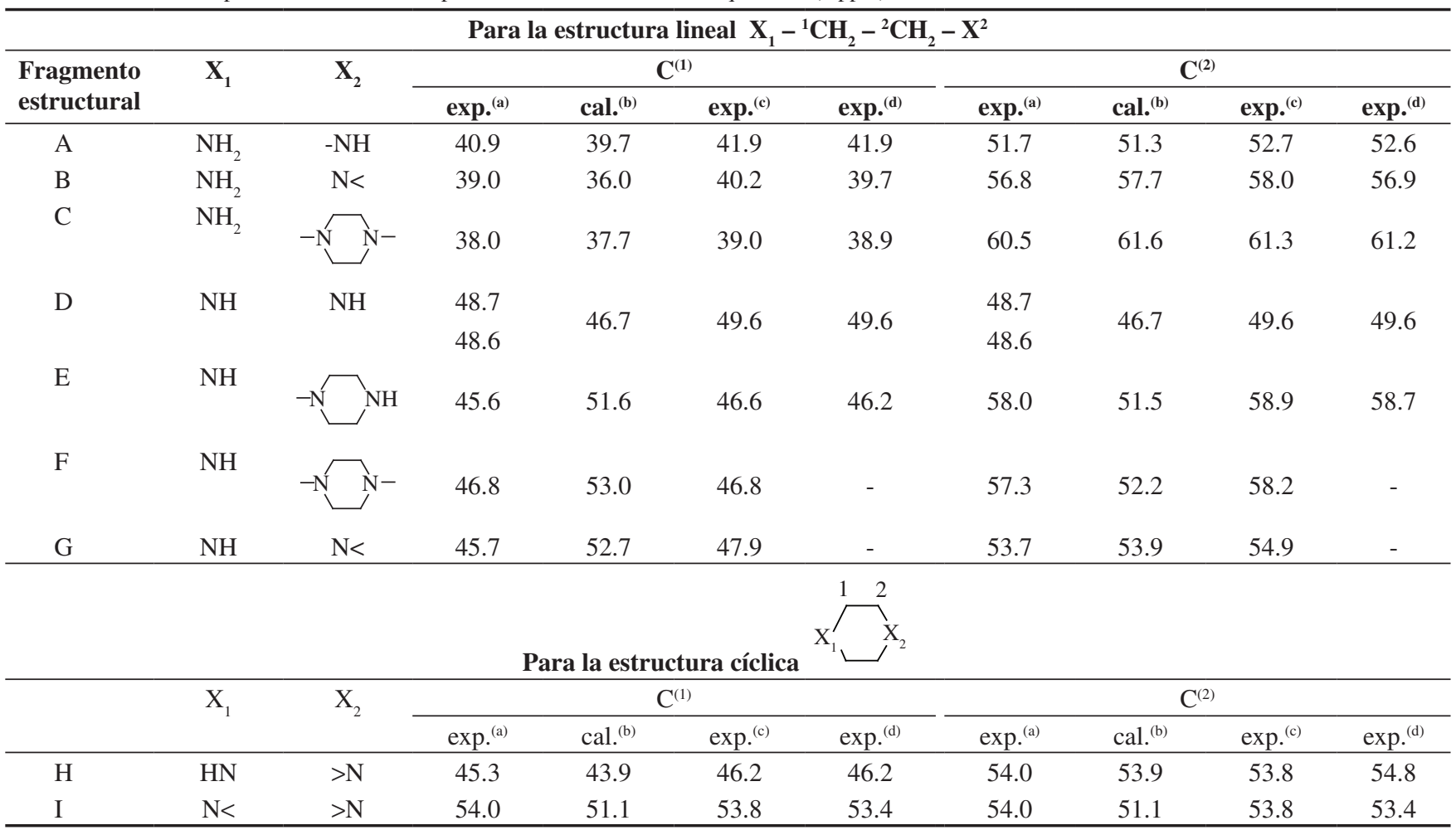

a) valores experimentales para el producto TEPA (ACROS, U.S.A.); b) valores calculados según la referencia 10; c) valores encontrados en la referencia 8; y d) valores encontrados en González Garcia et a ${ }^{[9]}$. 
Tabla 4. Composición cualitativa fundamental del producto TEPA.

$\begin{array}{lll}\text { 1) } & \mathrm{H}_{2} \mathrm{~N}-\left(\mathrm{CH}_{2}\right)_{2}-\mathrm{NH}-\left(\mathrm{CH}_{2}\right)_{2}-\mathrm{NH}-\left(\mathrm{CH}_{2}\right)_{2}-\mathrm{NH}_{2} & \begin{array}{l}\text { Trietilentetramina (TETA) } \\ \text { Tetraetilenpentamina (TEPA) } \\ \text { 3) }\end{array} \mathrm{H}_{2} \mathrm{~N}-\left(\mathrm{CH}_{2}\right)_{2}-\mathrm{NH}-\left(\mathrm{CH}_{2}\right)_{2}-\mathrm{NH}-\left(\mathrm{CH}_{2}\right)_{2}-\mathrm{NH}-\left(\mathrm{CH}_{2}\right)_{2}-\mathrm{NH}_{2} \\ \text { 4) } & \text { Aminoetiltris-aminoetilamina (AE-TAEA) } & \text { Aminoetildiaminoetilpiperazina (AE-DAEP) } \\ \mathrm{CH}_{2}-\mathrm{CH}_{2}-\mathrm{NH}_{2} & \\ \mathrm{H}_{2} \mathrm{~N}-\left(\mathrm{CH}_{2}\right)_{2}-\mathrm{NH}-\left(\mathrm{CH}_{2}\right)_{2}-\mathrm{N} \mathrm{CH}_{2}-\mathrm{NH}_{2} & \text { Aminoetilpiperazinoetilendiamina (AE-PEEDA) }\end{array}$

doblete puede relacionarse a la existencia de una mezcla de TETA y TEPA. No obstante, la mayor intensidad relativa de las señales atribuidas a la mezcla de éstos dos compuestos, cuando comparadas a las intensidades relativas de las otras señales del espectro pueden justificar la mayor concentración de estos dos compuestos en este producto (Figura 2). A pesar de estos argumentos, es esperado que el compuesto mayoritario sea la tetraetilenpentamina.

En el espectro de la Figura 2 fue evidente la presencia de cuatro nuevas señales en 46.8, 57.3, 45.7 y 53.7 ppm las que fueron asignadas a carbonos metilénicos de dos nuevos fragmentos estructurales como los mostrados en la Tabla 3 (fragmentos F y G). En la Tabla 4 son mostradas las moléculas consistentes con esos nuevos fragmentos estructurales, que corresponden a dos compuestos con estructuras semejantes a las encontradas anteriormente en el producto trietilentetramina (TETA), con un grupo etilenamina adicional $\left(>\mathrm{N}-\mathrm{CH}_{2}-\mathrm{CH}_{2}-\mathrm{N}<\right.$ ). Los otros compuestos propuestos en la composición del producto técnico TEPA son los siguientes:

En la Tabla 4 el compuesto 3 corresponde a otro de los compuestos propuestos en la composición de este producto. La estructura química de esta molécula es semejante a la molécula del compuesto tris-(2-aminoetil)amina (TAEA), que fue encontrado en la composición del producto comercial TETA (Tabla 2). La diferencia estructural entre estos dos compuestos consiste en la presencia de un grupo etilenamina adicional, que está enlazado químicamente a uno de los sustituyentes idénticos en el compuesto TAEA. Lo que origina que aparezca un nuevo fragmento estructural (fragmento G, Tabla 3) que contiene dos átomos de nitrógeno del tipo amina diferentes. Uno de los átomos de nitrógeno es secundario, y el otro átomo de nitrógeno es terciario $\left(-\mathrm{HN}-\mathrm{CH}_{2}-\mathrm{CH}_{2}-\mathrm{N}<\right)$. Este comportamiento estructural y los valores experimentales correspondientes a esta estructura química justifican la presencia del compuesto aminoetiltris-aminoetilamina (AE-TAEA) en este producto.

El otro nuevo fragmento estructural encontrado en el producto TEPA puede ser atribuido al compuesto 4 mostrado en la Tabla 4. Nos referimos al compuesto aminoetildiaminoetilpiperazina (AE-DAEP), donde la estructura cíclica de esta molécula es semejante a la del compuesto 4,4' aminoetilpiperazina (4,4' AEP), que fue propuesto en la composición del producto TETA (Tabla 2). En este caso, la molécula disubstituida de manera simétrica, cambia para no-simétrica. La diferencia estructural entre estas dos moléculas consiste en la presencia de un grupo etilenamina adicional, que está enlazado químicamente a uno de los sustituyentes idénticos en la estructura del compuesto 4,4' AEP. Lo que origina que aparezca un nuevo fragmento estructural (fragmento $\mathrm{F}$, Tabla 3), que contiene dos átomos de nitrógeno del tipo amina diferentes. Un de ellos es secundario y el otro es cíclico del tipo piperazina. Esta mudanza estructural y los valores experimentales correspondientes a esta estructura justifican la presencia del compuesto aminoetildiaminoetilpiperazina (AE-DAEP) en este producto.

Otro compuesto propuesto en la composición del producto técnico TEPA corresponde al compuesto 5 mostrado en la Tabla 4. Nos referimos a la aminoetilpiperazinoetilendiamina (AE-PEEDA) que corresponde a una molécula con estructura cíclica. Este compuesto es semejante a la estructura del compuesto dietilendiaminopiperazina (DEDAP), propuesto en la composición del producto TETA con un grupo etilenamina adicional (Tabla 2). En este caso, la molécula mono-substituida no cambia por la presencia de dos átomos metilénicos adicionales enlazados al único sustituyente de esta molécula. La presencia de este compuesto está justificada por los valores experimentales correspondientes a los fragmentos $\mathrm{H}, \mathrm{E}$, D y A mostrados en la Tabla 3.

Esta información relacionada a la presencia de cinco compuestos fundamentalmente en la composición de este producto técnico TEPA comercializado pela ACROS (U.S.A.), está de acuerdo con lo divulgado por la Compañía Química Dow con relación a la composición cualitativa de su producto semejante de tetraetilenpentamina (TEPA) (producto DEH 26) $)^{[2]}$.

La concentración de tetraetilenpentamina (TEPA) en el producto técnico de la ACROS (U.S.A.) fue de $55 \%$ molar. Este resultado fue obtenido a partir de la relación entre las áreas relativas de las señales en 40.9, 51.7 y 48.7 y 48.6 ppm, correspondientes a los carbonos metilénicos de los fragmentos estructurales del tipo, $-\mathrm{HN}-\mathrm{CH}_{2}-\mathrm{CH}_{2}-\mathrm{NH}_{2}$, $-\mathrm{HN}-\underline{\mathrm{CH}}_{2}-\mathrm{CH}_{2}-\mathrm{NH}_{2}$, y $-\mathrm{HN}-\underline{\mathrm{CH}}_{2}-\underline{\mathrm{CH}}_{2}-\mathrm{NH}-$, respectivamente en la estructura del compuesto TEPA, en relación a todas las áreas de las señales del espectro. Este valor de concentra- 
ción es aproximado, ya que está afectado por la presencia de trietilentetramina. Infelizmente ente caso el fabricante no informa el valor de la concentración de tetraetilenpentamina, lo que podría ser utilizado para fines comparativos. A pesar de esto, la Compañía Química Dow informa que el compuesto mayoritario en su producto semejante de TEPA (producto DEH 26) es el compuesto tetraetilenpentamina ${ }^{[2]}$.

\section{Conclusiones}

Los dos endurecedores comerciales de resinas epoxídicas del tipo etilenaminas comercializados por la ACROS (U.S.A.), fueron caracterizados cualitativamente utilizando la espectroscopia de resonancia magnética nuclear de carbono-13. En el producto trietilentetramina (TETA) el compuesto mayoritario fue TETA en una concentración de $60 \%$ molar, y en el producto tetraetilenpentamina (TEPA) el compuesto mayoritario fue TEPA en una concentración aproximada de 55\% molar. En los dos endurecedores estudiados en este trabajo se encontraron nueve fragmentos estructurales característicos de etilenaminas polifuncionales. Dos fragmentos lineales, dos fragmentos ramificados y cinco fragmentos cíclicos del tipo piperazina. La composición cualitativa encontrada en estos dos productos fue semejante a la composición divulgada para los productos similares TETA y TEPA comercializados por la Compañía Química Dow, productos DEH 24 y DEH 26, respectivamente.

\section{Agradecimientos}

Los autores agradecen el apoyo del Plano Nacional de Ciencia y Tecnología del Sector Petróleo y Gas Natural - CT-
PETRO, por medio de CNPq (CT-PETRO/CNPq) Proceso No. 500092/02-8.

\section{Referencias Bibliográficas}

1. Lee, H. \& Neville, K. - "Handbook of Epoxy Resins", cap.7, McGraw-Hill, Inc., New York (1967).

2. Dow Chemical Company, www.dow.com

3. Gonzalez Garcia F.; da Silva, P. M.; Soares, B. G. \& Rieumont, J. - Polymer Testing, 26, p.95 (2007).

4. González Garcia, F.; Miguez, E. \& Soares, B. G. - Polímeros: Ciência e Tecnologia, 15, p.261 (2005).

5. Bauer, P. \& Richter, M. - Journal of Chromatography, 206, p.343 (1981).

6. Henriks-Eckerman, M. \& Laijoki, T. - Journal of Chromatography, 333, p.220 (1985).

7. Winkler, E. Hohaus, E. \& Felber, E. - Journal of Chromatography, 436, p.447 (1988).

8. Bulai, A. K.; Slonim, I. Y.; Urman, Y. G.; Vakulenko, V. A. \& Chetverikova, A. T. - Polymer Science U.S.S.R., 28, p.1854 (1986).

9. González Garcia, F.; Pérez, C.; Galego, N.; Martínez, R. \& Rosado, A. - Revista Cubana de Química, V, p.44 (1989).

10. Prestch, E. \& Buhlman, P. - "Structure Determination of Organic Compounds: Tables of Spectral Data", $3^{\circ}$ ed Springer (2004).

Enviado: 08/05/07

Reenviado: 28/07/07

Aceito: 14/08/07 